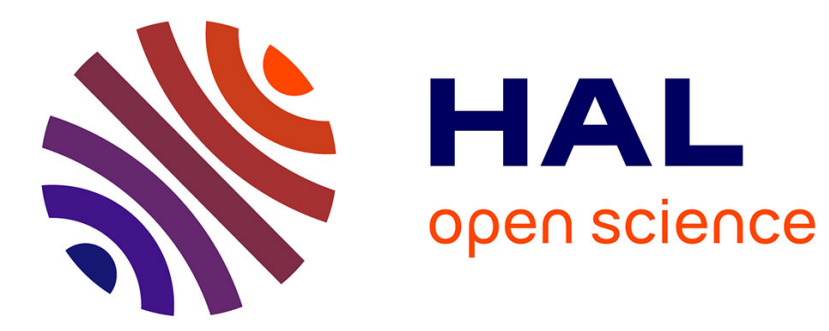

\title{
Current understanding of cellulose ethers impact on the hydration of C3A and C3A-sulphate systems
}

\author{
Jérémie Pourchez, Philippe Grosseau, Bernard Ruot
}

\section{To cite this version:}

Jérémie Pourchez, Philippe Grosseau, Bernard Ruot. Current understanding of cellulose ethers impact on the hydration of C3A and C3A-sulphate systems. Cement and Concrete Research, 2009, 39 (8), pp.664-669. 10.1016/j.cemconres.2009.05.009 . hal-00409937

\section{HAL Id: hal-00409937 https://hal.science/hal-00409937}

Submitted on 14 Aug 2009

HAL is a multi-disciplinary open access archive for the deposit and dissemination of scientific research documents, whether they are published or not. The documents may come from teaching and research institutions in France or abroad, or from public or private research centers.
L'archive ouverte pluridisciplinaire HAL, est destinée au dépôt et à la diffusion de documents scientifiques de niveau recherche, publiés ou non, émanant des établissements d'enseignement et de recherche français ou étrangers, des laboratoires publics ou privés. 


\section{Current understanding of cellulose ethers impact on the hydration of C3A and C3A-sulphate systems}

\section{J. Pourchez (1*), P. Grosseau (1), B. Ruot (2)}

(1) Ecole Nationale Supérieure des Mines de Saint-Étienne (ENSMSE), Centre SPIN, LPMG: process in granular media laboratory CNRS UMR 5148, 158, cours Fauriel 42023 Saint-Étienne cedex 2 - France

(2) Scientific and Technical Centre for Building (CSTB) 24, rue J oseph Fourier - 38400 Saint-Martin-d'Hères - France

(*) pourchez@emse.fr

\section{Abstract.}

The impact of cellulose ethers (CE) on C3A hydration was examined to support the understanding of the retarding effect of $\mathrm{CE}$ on cement hydration. In this sense, we successively studied the CE adsorption on ettringite and calcium hydroaluminates, and then the $\mathrm{CE}$ influence during C3A hydration in presence or absence of calcium sulphate. We emphasized a phase-specific adsorption of $\mathrm{CE}$ depending on $\mathrm{CE}$ chemistry. Besides, in addition of $\mathrm{CE}$, we highlighted a gradual slowing down of C3A dissolution as well as ettringite and calcium hydroaluminates precipitation. Again, a great impact of CE chemistry and CE adsorption behaviour were noticed. Thus, HECs induce always a stronger adsorption on calcium hydroaluminates and a longer C3A hydration delay that HPMCs.

Keywords:

Cellulose ethers; Hydration; Adsorption; C3A; Calcium sulphate.

\section{1- Introduction}

Cellulose ethers (CE) are commonly introduced into factory-made mortar formulations in order to induce a substantial increase of water retention capacity as well as to improve the workability of fresh material as well as the adherence to the substrate. Hence, these molecules contribute to good mechanical strength of the hardened material. However, as a side effect, $\mathrm{CE}$ may also induce a more or less important slowing down of cement hydration. Preliminary studies highlight that there was no significant degradation of CE [1]. Therefore, the mechanism of hydration delay induced by CE cannot be explained thanks to the impact of hydroxy carboxylic acids generated during their alkaline degradation. Moreover, recent insights emphasize a great influence of CE during C3S hydration [2]. Firstly, it seems that the influence of $\mathrm{CE}$ on C3S dissolution kinetic was quite negligible. All things considered, it was assumed that CE strongly act on C-S-H precipitation by means of: a decrease of the amount of initial C-S-H nuclei, a delay in order to obtain a continuous C-S-H shell around the C3S grain, and finally a formation of a thicker and more permeable C-S-H layer.

The tricalcium aluminate (C3A) is one of the most reactive major components of clinker. C3A strongly reacts with water to form calcium hydroaluminates (i.e. Afm-type phase and the more thermodynamically stable hydrogarnet), whereas addition of calcium sulphate leads to the precipitation of ettringite with a slower rate. On further examination, the presence of sulphates allows to regulate the kinetics of C3A hydration to avoid the "flash-setting" of cement. Thus, the reactivity of C3A-sulphate system appears crucial so as to control and predict the workability of fresh cement material. But mechanisms and parameters controlling the reactivity of C3A hydration are not yet fully elucidated, even if recent work significantly improve our knowledge on this topic [3, 4]. Besides, very few studies dealt with the hydration behaviour of C3A in presence of CE. Using soft X-ray transmission microscopy, Silva underlined that CE may slightly affect the aspect of C3A hydration [5]. It was observed that 
the single CE molecule studied lead to retard the kinetics of C3A hydration from the few minutes after mixing. However, Silva does not succeed from these microscopic images to state whether the delay is mainly caused by $\mathrm{CE}$ adsorption onto cementitious grains, by slowing down ionic diffusion or by chemical interactions.

Thus, this paper is devoted to determine the influence of $\mathrm{CE}$ chemistry on the kinetics and mechanism of C3A hydration. We studied increasing complexity mineral systems (pure C3A admixed or not, and then C3A-sulphate system admixed or not) in order to bring original experimental data as well as to support a sound knowledge on CE-C3A interactions. In this context, to follow an analytic approach, we successively examined the CE adsorption on ettringite and calcium hydroaluminates, as well as the influence of $\mathrm{CE}$ during C3A hydration in different systems.

\section{2- Materials and methods}

\section{1- Mineral compounds}

The investigated mineral compounds were supplied by Lafarge and Italcementi companies. The pure anhydrous or hydrated phases were prepared at laboratory scale, with the help of industrial partners. C3A was obtained by burning the appropriate amount of calcium carbonate and alumina, whereas the ettringite was synthesized from calcium oxide and aluminium sulphate 16-hydrate. Several characterisations were carried out in order to quantify the phase assemblage of the investigated mineral systems. X-ray diffraction (XRD) analysis (Siemens, D 5000, Germany) allowed to quantify the phase composition by means of Rietveld method (Siroquant V2.5 software).

\section{2- Organic products}

Among the wide variety of existing $\mathrm{CE}$, three types are mainly used in mortar manufacturing: hydroxypropylmethyl cellulose (HPMC), hydroxyethylmethyl cellulose (HEMC) and hydroxyethyl cellulose (HEC). The chemical structure of HEC is entirely determined by two parameters i.e. the molecular weight (Mw) and the hydroxyethyl content $(\% \mathrm{OC} 2 \mathrm{H} 4 \mathrm{OH})$. Conversely, HPMC and HEMC are determined by three structural parameters i.e. the molecular weight, the methoxyl content (\% OCH3), and the hydroxypropyl (in HPMC, $\% \mathrm{OC} 3 \mathrm{H} 6 \mathrm{OH}$ ) or hydroxyethyl (in $\mathrm{HEMC}, \% \mathrm{OC} 2 \mathrm{H} 4 \mathrm{OH}$ ) content.

Besides, a CE molecule was also determined by two structural parameters named DS and MS. Indeed, the amount of substituent groups on the anhydroglucose units can be designated by weight percent or by the average number of substituent groups attached to the ring, a concept known to cellulose chemists as "degree of substitution" noted DS. The DS corresponds to the average number of hydroxyl groups per anhydroglucose unit rings substituted by etherifying groups to become a hydroxyethyl, hydroxypropyl or methoxyl group. The molar substitution (MS) reports the number of moles of hydroxypropyl groups per mole of anhydroglucose.

The molecular weight (Mw) distribution was performed by Size Exclusion Chromatography (SEC) using 8 polymaltotrioses as standard samples. A very minor population of oligomers $(\mathrm{Mw}<500 \mathrm{Da})$ is only detected for HECs. The substitution degree was investigated by Near Infra-Red spectroscopy (NIR). All details concerning SEC and NIR protocols were previously described [6]. CE characterization results are detailed in Table 1.

\section{3- Brief overview of the experimental methods}

In this paper, we applied a similar experimental methodology that the approach proposed for the study of CE-C3S interactions. As a result, steps of each protocol are precisely detailed in a previous paper [2]. In short, we can just recall the main items of the experimental techniques. Firstly, adsorption data of $\mathrm{CE}$ on aluminate phases was based on the dosage of $\mathrm{CE}$ into supernatant thanks to a phenol-sulfuric acid method. The concept is to put in contact a given [CE] with a well-characterized mineral phase. After 2 hours of exposure time, under nitrogen atmosphere and constant magnetic stirring, [CE] in the supernatant was determined. 
The amount of polymer adsorbed on the mineral phase was finally easily calculated [2]. Besides, conductometry is a powerful tool for monitoring C3A hydration kinetics because of this method provides easily rather detailed information on the different steps of the hydration reaction [3, 4]. In fact, conductometry appears as a convenient comparative method as long as the relative concentrations of all ingredients are kept constant and only one variable is changed at a time (in this case, the chemistry of $\mathrm{CE}$ ). C3A hydration was performed in a limewater solution $([\mathrm{Ca}(\mathrm{OH}) 2]=20 \mathrm{mM})$ with a liquid to solid ratio equal to 20 . The surface area of the mineral phases was analyzed by nitrogen adsorption in a Micromeritics ASAP 2000 nitrogen adsorption apparatus. The samples measured were degassed at $100{ }^{\circ} \mathrm{C}$ before the measurements. The surface area was determined by the multipoint BET method using the adsorption data. Results are reported in Table 2. The determination of calcium, aluminium and sulphate concentration was obtained using ionic chromatography. This was performed on a Dionex apparatus composed of a GP50 pump, a CS12A column for cation analysis, a AS11HC column for anions, a CD conductometric detector and an UV-visible detector. The analysis conditions are given in [2].

\section{3- Adsorption of cellulose ethers on ettringite and calcium hydroaluminates}

As C3A reacts instantaneously with water to form calcium hydroaluminates, it is impossible to quantify the adsorption of $\mathrm{CE}$ on only C3A surface. On the contrary, we assume that the hydrated pure phases (i.e. ettringite and calcium hydroaluminates) were in quite stable equilibrium with the liquid phase. In this sense, in order to limit as far as we can any solid dissolution, adsorption experiments were performed with a solution previously ionsaturated in respect of each mineral phase investigated. We must also underline that, while ettringite is a pure phase synthesized at laboratory scale, the "calcium hydroaluminates" sample is simply obtained by hydrating pure C3A in water (without gypsum, liquid to solid ratio equal to 20) during 1 hour. We followed the C3A hydration by conductometry, and then we characterized the solid by SEM (Fig. 1), XRD and BET measurement. All results emphasize that C2AH8 - C4AH13 but also C3AH6 hydrogarnet structure, are the major phases composing this sample called "hydroaluminates".

The content of CE adsorbed was quantified per mass of mineral phase introduced (Fig. 2). A very disparate CE-adsorption behaviour is noticed as a function of the hydrated aluminate phases. In fact, whereas an important CE adsorption on calcium hydroaluminates is observed, no significant adsorption on ettringite is emphasized. In other words, CE adsorption appears phase-specific or more rigorously the considered phases (ettringite versus "hydroaluminates") are CE-adsorption specific. Moreover, a great impact of $\mathrm{CE}$ chemistry on calcium hydroaluminates adsorption is highlighted. Obviously, HECs (i.e. H1, N1 and N7) show an elevated adsorption on calcium hydroaluminates always higher that 75 wt \%, although HPMCs (i.e. U2 and P1) induce a weak adsorption always lower that 20 wt \%. Finally, the surface area of mineral phases was also determined prior to experiment (Table 2). This leads to determine the content of $\mathrm{CE}$ adsorbed per square meter of calcium hydroaluminates introduced. We note that HECs adsorption on calcium hydroaluminates is always higher to $200 \mu \mathrm{g} / \mathrm{m} 2$ although HPMCs adsorption is around $50 \mu \mathrm{g} / \mathrm{m} 2$. HECs adsorption on calcium hydroaluminates appears at least 4 times higher that HPMCs adsorption. All things considered, we demonstrate that significant $\mathrm{CE}$ adsorption can occurs and markedly depends on both CE chemistry and substrate phase (i.e. calcium hydroaluminates or ettringite).

\section{4- Hydration of pure C3A in diluted limewater}

We began with the study of the non admixed pure C3A in diluted media. The reaction was monitored by conductometry and ionic chromatography (Fig. 3). We emphasized that the conductivity curve perfectly reflects the calcium concentration evolution. Furthermore, the aluminium concentration evolves in the opposite way than the calcium concentration. The conductivity drop observed can be induced by the amphoteric nature of aluminium ion acting 
as an acid and thus forming aluminate ions by depleting the hydroxide concentration. Observably, three different stages were distinguished (Fig. 3).

The first stage corresponds to the decrease of the conductivity. In the same time, the calcium concentration decreases while the aluminium concentration increases. XRD experiment was also performed in order to identify phases which successively precipitated. According to the literature [3,7], we assume that this first stage highlights C3A dissolution with simultaneously calcium hydroaluminates precipitation. The calcium to aluminium ratio is equal to 1.5 during C3A dissolution. As the calcium concentration decreases while the aluminium concentration increases, it appears that the calcium to aluminium ratio of calcium hydroaluminates which precipitate should be higher than 1.5. Minard proposed in order to explain the different stoechiometry of calcium hydroaluminates that a solid solution CxAHy probably exists in which C2AH8 and C4AH13 are two limits [7].

During the second stage, a plateau is observed for all parameters monitored: conductivity, aluminium and calcium concentration. This indicates that a steady-state of C3A hydration reaction occurs. Therefore, hydration products which precipitate during this period have globally a calcium to aluminium ratio very close to the one releases during C3A dissolution, i.e. 1.5. Moreover, any hydrogarnet C3AH6 was detected by XRD during this second stage. As a result, the stoechiometric ratio of 1.5 corresponds to the precipitation of one mole of C2AH8 and one mole of $\mathrm{C} 4 \mathrm{AH} 13$, for the dissolution of 2 moles of $\mathrm{C} 3 \mathrm{~A}$.

Finally, XRD experiments put clearly in evidence the precipitation of hydrogarnet C3AH6 during the third stage. The precipitation of C3AH6 induces an increase of the calcium concentration (to reach a plateau) and the progressive consumption of all aluminium ions of the solution.

\section{5- Influence of cellulose ethers on C3A hydration in absence of gypsum}

We examined the C3A hydration in limewater without calcium sulphate and in addition of $\mathrm{CE}$. So as to exhibit how $\mathrm{CE}$ can act during calcium hydroaluminates precipitation, we focused on HEC H1 which induces the stronger slowing down behaviour. The monitoring of the conductivity as well as calcium and aluminium ions allowed to bring interesting data. More precisely, two main stages were clearly put in evidence during C3A hydration without calcium sulphate and in presence of H1 (Fig. 4). During the first stage we observed a slower rate of calcium ions decrease (Fig. 4), in comparison with pure C3A hydration without calcium sulphate and CE (Fig. 3). Furthermore, the rise of aluminium concentration reaches 10 $\mathrm{mmol} / \mathrm{L}$ at the end of the first stage (Fig. 4), a value 5 times higher that the maximum of aluminium concentration attained in the case of pure C3A hydration without calcium sulphate and CE (Fig. 3). This indicates both a slowing down of C3A dissolution and calcium hydroaluminates precipitation. Besides, as the calcium concentration decreases whereas the aluminium concentration rises, we assume that the calcium to aluminium ratio of calcium hydroaluminates which precipitate should be higher than 1.5. Finally, when the first period is finished, even if the hydroaluminates precipitation is doubtless put in evidence by SEM observation of C3A grain, the amount of calcium hydroaluminates is not enough to be detected by XRD. Then, the beginning of the second period corresponds to the start of the aluminium concentration reducing while the calcium concentration remains constant. Thus, we assume that this second period highlights a very progressive C3AH6 precipitation (confirmed by XRD characterization). To sum up, H1 doubtless induces a strong slowing down of both C3A dissolution and calcium hydroaluminates precipitation.

Afterwards, we showed that the slowing down of C3A hydration hardly depends on the chemical nature of the CE applied (Fig. 5). In fact, a gradual effect on the delay was doubtless observed as a function of the type of CE molecule introduced. Obviously, we noticed that HECs (i.e. H1, N1 and N7) induce a stronger slowing down that HPMCs (i.e. U2 and P1). Thus, we demonstrated that CE chemistry plays a major role on the retardation of C3A hydration without calcium sulphate. Moreover, we noticed that the higher the $\mathrm{CE}$ adsorption on calcium 
hydroaluminates (Fig. 2), the stronger the delay of the precipitation of calcium hydroaluminates (Fig. 5).

All things considered, we perfectly showed that CE can induced a slowing down of both C3A dissolution and calcium hydroaluminates precipitation. This delay seems to be mainly caused by $\mathrm{CE}$ adsorption, on at least hydroaluminates, because of the higher the $\mathrm{CE}$ adsorption on calcium hydroaluminates (Fig. 2), the stronger the delay of the precipitation of calcium hydroaluminates (Fig. 5). As a result, CE chemistry appears as an important controlling factor since we noticed that the retardation of C3A hydration without calcium sulphate is well-correlated to the CE-adsorption capacity on calcium hydroaluminates while this CE-adsorption mainly depends on CE structural parameters.

\section{6- Hydration of C3A-gypsum system in diluted limewater}

Firstly, we studied the hydration behaviour of the non admixed C3A-gypsum system in diluted media. The solution conductivity as well as calcium, aluminium and sulphate concentrations were followed during the conductometry experiments. Two periods were clearly observed (Fig. 6).

The first hydration period of C3A-gypsum system corresponds to the presence of sulphate ions into solution. Based on different works [3,4,7], this period refers to gypsum and C3A dissolution where as the ettringite formation takes place. During this first stage we notice a continuously decrease of calcium ions while no aluminium ions are detected into solution. Moreover, the sulphate concentration rises until a plateau at the beginning of the reaction, despite the fact that these ions were consumed to precipitate ettringite. This plateau is very short (around 5 minutes) when only 2 wt \% of gypsum is initially added to C3A. In fact, although sulphate ions were consumed by the reaction to form ettringite, they were instantaneously replaced by the dissolution of gypsum to obtain a quite constant concentration of sulphate into solution. This quasi-steady state lasts as long as gypsum dissolution occurs, i.e. as long as solid gypsum remains in solution. Hence, the higher the initial amount of gypsum added initially to C3A, the longer the duration of this period during which the gypsum dissolution occurs. Therefore we can easily increase the duration of the quasi-steady state, between gypsum dissolution and ettringite precipitation, by increasing the initial amount of calcium sulphate added to C3A (Fig. 7). An initial linear evolution of conductivity, sign of the quasi-steady state instatement, is clearly highlighted from an addition of $10 \mathrm{ut} \%$ of gypsum. At the end of this linear evolution, or in other words when no more solid gypsum is available, the sulphate concentration starts to decrease because of ettringite precipitation. From this time, a slightly decrease of conductivity and sulphate ions is noticed. That means that sulphate ions consumed in order to precipitate ettringite only come from sulphate ions previously stored up into solution during the gypsum dissolution. To summarize, during the first period of C3A-gypsum hydration corresponding to the presence of sulphate ions into solution, two main steps of ettringite precipitation is distinguished depending of the presence or not of solid gypsum.

The second hydration period of C3A-gypsum system starts when sulphate ions are entirely consumed. In fact, at the beginning of this second period, a fall of both conductivity and calcium ions occurs. In the same time, the sulphate concentration reaches zero, while aluminium ions start to appear into solution. According to Minard works [3,7], these observations obviously emphasize that, when the ettringite precipitation stops because of the exhaustion of sulphate ions, a huge calcium hydroaluminates precipitation takes place. In fact, the reactions observed simply match to the hydration of C3A in limewater without gypsum. Thus, the second period corresponds to the C3A dissolution and the calcium hydroaluminates precipitation, which is at the origin of the strong drop of conductivity since consuming calcium ions and releasing aluminium ions into solution. 


\section{7- Influence of cellulose ethers on C3A hydration in presence of gypsum}

To improve our understanding of the mechanism at the origin of this slowing down behaviour induced by $\mathrm{CE}$, we studied $\mathrm{H} 1$ admixed C3A-gypsum system. We firstly focused on a system containing 2 wt\% of gypsum. Three stages were discriminated (Fig. 8).

* The first stage corresponds to the ettringite precipitation from sulphate ions of the solution without any gypsum dissolution. This part of the hydration is similar with the non admixed system (Fig. 7). However, the time necessary to consume all sulphate ions is 10 minutes higher compared to the non admixed system. Therefore, we assumed that CE may induce a weak slowing down of C3A dissolution and ettringite precipitation when all the calcium sulphate is dissolved.

* When sulphate ions are exhausted, although the instantaneous and massive precipitation of calcium hydroaluminates should happen, conversely we emphasized a rise of calcium concentration and conductivity. In fact, it seems that the calcium hydroaluminates precipitation is blocked. In presence of $\mathrm{CE}$, the system seems to need a higher critical supersaturation level in order to precipitate calcium hydroaluminates. As there is no more sulphate to form ettringite, the only reaction which can happen is C3A dissolution. So, during this second stage, we observed only the C3A dissolution so as to reach a higher critical supersaturation with respect to calcium hydroaluminates. Moreover, as this second period reflects only the C3A dissolution, the slope of the conductivity curve during this stage is directly proportional to the rate of C3A dissolution. As a result, on the figure 9, we highlighted that the stronger the delay on both ettringite precipitation and C3A dissolution (i.e. the longer the duration to consume all sulphate ions), the slower the C3A dissolution rate (i.e. the lower the conductivity slope during the second stage where only C3A dissolution occurs).

* Finally, when the critical supersaturation of the liquid phase is enough elevated, the conductivity and calcium ions decrease while aluminium concentration starts to rise. Similarly to the second period of the non admixed C3A-gypsum system, this third stage of $\mathrm{H} 1$ admixed C3A-gypsum system indicates a precipitation of calcium hydroaluminates. However, the decrease of the conductivity slope and calcium concentration is weak in comparison with the non admixed system (Fig. 7). Thus, the rate of calcium hydroaluminates precipitation seems to be significantly slowing down in presence of $\mathrm{H} 1$.

The impact of CE chemistry in the hydration of C3A-gypsum system in diluted limewater was also performed using different gypsum to C3A weight ratio equal to 2 and 10 ut \% (Fig. 9 and 9). Results highlight a gradual effect on the slowing down of both ettringite and calcium hydroaluminates precipitation. The CE chemistry seems to play a major role since whatever the gypsum ratio added. Noticeably, we always remarked that HECs (i.e. H1, N1 and N7) induce a stronger slowing down that HPMCs (i.e. U2 and P1).

On the one hand, we examined the influence of $\mathrm{CE}$ on ettringite precipitation. In presence of 10 wt \% of gypsum (Fig. 10), we can discriminate the two steps of ettringite precipitation previously described in paragraph 6. Conversely, in presence of 2 ut \% of gypsum (Fig. 9), we only distinguish the second step of ettringite precipitation (i.e. the ettringite precipitation thanks to sulphate ions coming from the solution), because of the small amount of gypsum initially added to C3A is quickly dissolved during the first minutes of hydration. Besides, during the first step (i.e. the pseudo steady-state instatement where all sulphate ions are consumed by ettringite formation are replaced into solution by gypsum dissolution) no significant impact of CE is put in evidence. However, whatever the gypsum ratio added, a strong impact of $\mathrm{CE}$ is emphasized during the second step of ettringite precipitation. Observably, the slope of conductivity curves during the second step of ettringite precipitation as well as the duration to reach the beginning of the second period of hydration (i.e. the 
duration to reach the time where all sulphate ions are consumed) is more important in presence of CE (Fig. 9 and 10). In other words a significant slowing down of both ettringite precipitation and C3A dissolution rate, depending on CE chemistry, is perceived. This delay occurs whatever the gypsum ratio added, but CE act only during the second step of ettringite precipitation (i.e. where the solid gypsum are entirely dissolved). It was also very interesting to underline that $\mathrm{CE}$ can reduce the ettringite precipitation rate although no significant adsorption of CE on ettringite was observed (Fig. 2).

On the other hand, we also studied the influence of $\mathrm{CE}$ on calcium hydroaluminates precipitation during the second hydration period of C3A-gypsum, when sulphate ions are exhausted. In coherence with results illustrated in paragraph 5, a more or less slowing down of calcium hydroaluminates is emphasized as a function of CE chemistry. For HPMCs admixed system, we observe a strong drop of conductivity, sign of a massive and instantaneous precipitation of calcium hydroaluminates (Fig. 9 and 10) comparable to non admixed system. On the contrary, for HECs admixed system, we highlight a slower rate of calcium hydroaluminates precipitation. Moreover, it appears that the stronger the CEadsorption on calcium hydroaluminates (Fig. 2), the stronger the slowing down of calcium hydroaluminates (Fig. 9).

\section{8- Conclusion}

Significant slowing down of C3A hydration is put in evidence in diluted media. In particular, very low molecular mass $\mathrm{CE}$ (e.g. $\mathrm{H} 1$ molecule) are enable to strongly delay the C3A hydration although no rise of viscosity is observed regarding to the high L/S used. As a result, all things points to say that the retardation caused by $\mathrm{CE}$ cannot be explained thanks to ionic motion hindrance assumption.

Furthermore, we demonstrate that CE adsorption is phase-specific. As a matter of fact an important CE adsorption on calcium hydroaluminates is emphasized, while no significant adsorption on ettringite is noticed. Moreover a great impact of CE chemistry on calcium hydroaluminates is highlighted because of HECs adsorption on this phase appears at least 4 times higher that HPMCs adsorption.

CE lead also to a gradual slowing down of the C3A hydration depending on CE chemistry. For all experimental conditions, we remark that HECs induce a higher delay that HPMCs. Based on these observations, substitutions groups (nature and content) seems to be more important controlling factors on C3A hydration rather than molecular mass. In fact, without calcium sulphate, we show that CE can reduce both the C3A dissolution as well as the calcium hydroaluminates precipitation. It seems that the higher the $\mathrm{CE}$ adsorption on calcium hydroaluminates, the stronger the slowing down of calcium hydroaluminates precipitation. In addition of calcium sulphate, results emphasized that whatever the amount of gypsum added, $\mathrm{CE}$ induce a weak slowing down of C3A dissolution, ettringite precipitation and then a strong reduce of calcium hydroaluminates precipitation.

\section{Acknowledgements}

The authors would like to acknowledge the financial support of the international CEReM network (consortium for study and research on mortars - http:// cerem.cstb.fr), and many helpful conversations with industrial and academic CEReM partners. We also extend to thank Pr. A. Nonat, from the Burgundy University, for fruitful discussions and comments which greatly contribute to this paper. 


\section{References}

[1] J. Pourchez, A. Govin, P. Grosseau, R. Guyonnet, B. Guilhot, B. Ruot, Alkaline stability of cellulose ethers and impacts of their degradation products on cement hydration, Cem. Concr. Res. 36 (2006) 1252-1256.

[2] J. Pourchez, P. Grosseau, B. Ruot, Changes in C3S hydration in presence of cellulose ethers, Cem. Concr. Res. XX under review.

[3] H. Minard, S. Garrault, L. Regnaud, A. Nonat, Mechanisms and parameters controlling the tricalcium aluminate reactivity in the presence of gypsum, Cem. Concr. Res. 37 (2007) 1418-1426.

[4] A. Lagosz, J. Malolepszy, S. Garrault, Hydration of tricalcium aluminate in the presence of various amounts of calcium sulphite hemidrate: conductivity tests, Cem. Concr. Res. 36 (2006) 1016-1022.

[5] D.A. Silva, P.J .M. Monteiro, The influence of polymers on the hydration of portland cement phases analyzed by soft X-ray transmission microscopy, Cem. Concr. Res. 36 (2006) 1501-1507.

[6] J. Pourchez, A. Peschard, P. Grosseau, B. Guilhot, R. Guyonnet, F. Vallee, HPMC and HEMC influence on cement hydration, Cem. Concr. Res. 36 (2006) 288-294.

[7] H. Minard, A. Nonat, S. Garrault, Understanding of reactional sequences and limiting stages during tricalcium aluminate hydration with and without gypsum, Proceeding of the 12th International Congress on the Chemistry of Cement, Montréal (2007).

Tables

\begin{tabular}{|c|c|c|c|c|c|c|}
\hline Admixtures & $\begin{array}{r}\text { Mw } \\
\text { (dalton) }\end{array}$ & $\begin{array}{c}\mathrm{OC} 2 \mathrm{H} 4 \mathrm{OH} \\
(\%)\end{array}$ & $\begin{array}{l}\mathrm{OC} 3 \mathrm{H} 6 \mathrm{OH} \\
(\%)\end{array}$ & $\begin{array}{l}\text { OCH3 } \\
(\%)\end{array}$ & $S^{D}$ & $\mathrm{~S}^{\mathrm{M}}$ \\
\hline HPMC U2 & 955000 & - & 10.65 & 27.5 & 1. & 0 \\
\hline HPMC P1 & 175000 & - & 19 & 27.5 & $\begin{array}{c}82 \\
1 . \\
95\end{array}$ & $\begin{array}{r}.29 \\
0 \\
.56\end{array}$ \\
\hline HEC H1 & 175000 & 48.5 & - & - & - & 2 \\
\hline HECN1 & 175000 & 56 & - & - & - & 2 \\
\hline HEC N7 & $\begin{array}{l}1335 \\
000\end{array}$ & 56 & - & - & - & $\begin{array}{c}.5 \\
2 \\
5\end{array}$ \\
\hline
\end{tabular}

Table 1. Molecular parameters of the selected cellulose ethers.

\begin{tabular}{c|c} 
Mineral phases & $\begin{array}{c}\text { Surface area } \\
\text { (m2.g-1) }\end{array}$ \\
\hline C3A & $0.51 \pm 0.01$ \\
Gypsum & $0.53 \pm 0.02$ \\
\hline $\begin{array}{c}\text { Ettringite } \\
\text { C3A hydrated 30 min } \\
\text { (called "hydroaluminates") }\end{array}$ & $7.79 \pm 0.07$ \\
\hline
\end{tabular}

Table 2. Surface area measured of the mineral phases studied. 


\section{Figures}
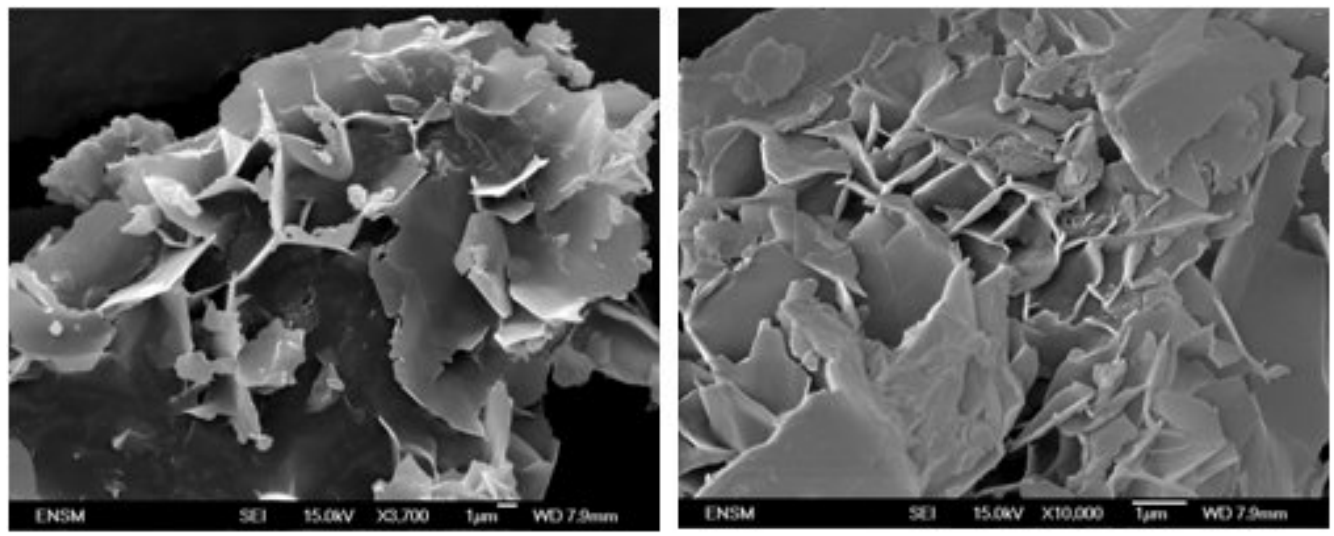

Figure 1. SEM observation of calcium hydroaluminates faceplate after C3A grain hydration during 1 hour in water (liquid to solid ratio equal to 20)

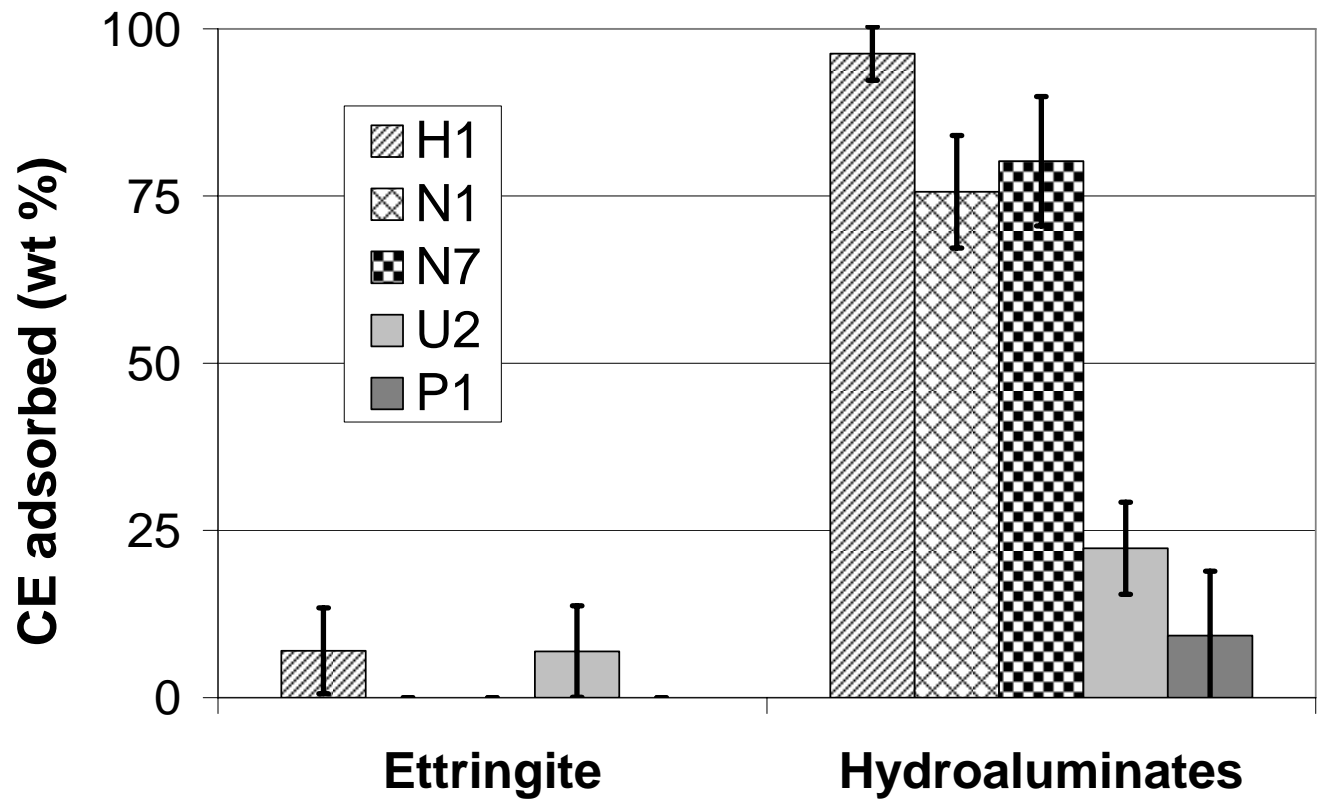

Figure 2. CE adsorption on ettringite and hydroaluminates expressed in wt $\%$ after a 2 hour-exposure time (initial concentration of $\mathrm{CE}$ equal to $15 \mathrm{mg}$ per $\mathrm{g}$ of pure mineral phase introduced, $\mathrm{L} / \mathrm{S}=20$ ). 


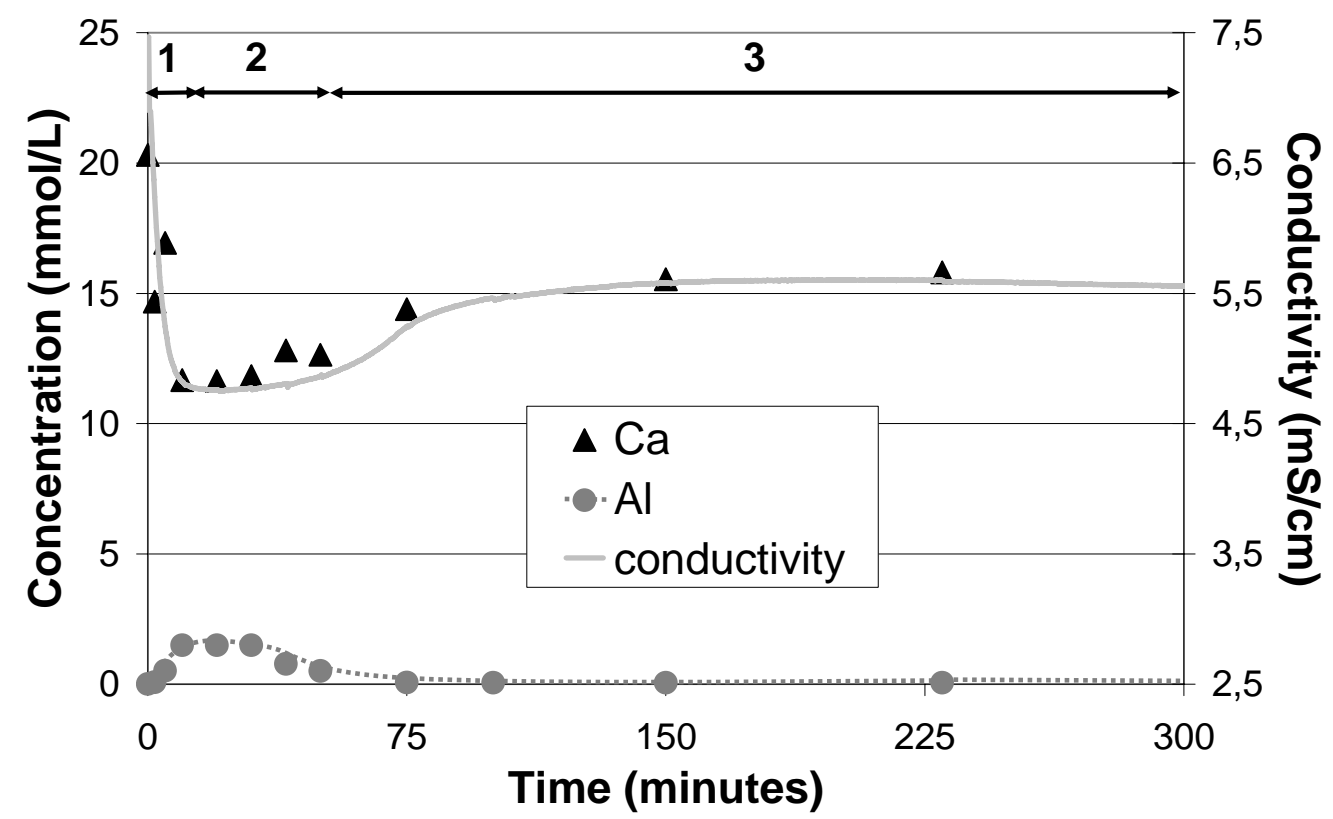

Figure 3. C3A hydration in limewater without calcium sulphate: conductivity curve, calcium and aluminium concentration ( $\mathrm{L} / \mathrm{S}=20,[\mathrm{Ca}(\mathrm{OH}) 2=20 \mathrm{mM}])$.

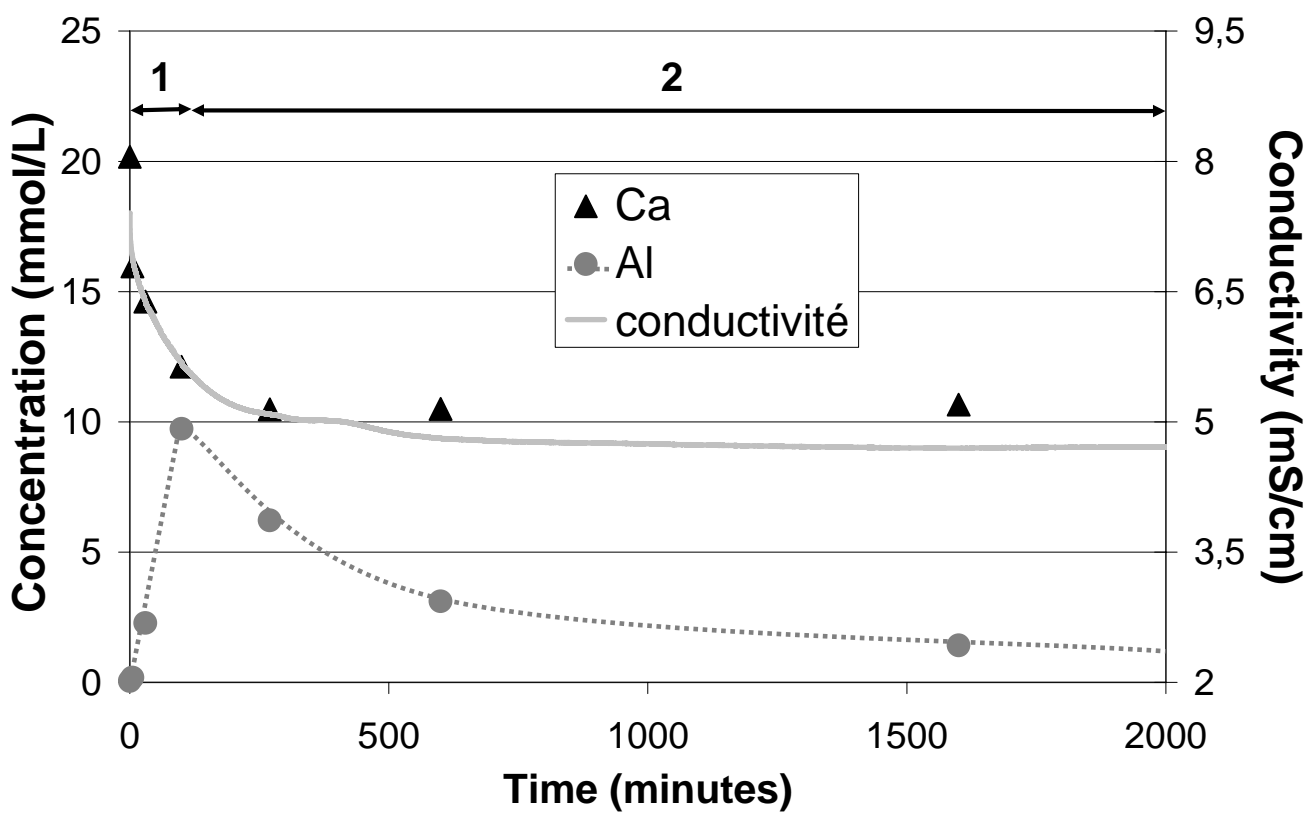

Figure 4. Impact of HEC H1 on C3A hydration in limewater without calcium sulphate: conductivity curve, calcium and aluminium concentration $(\mathrm{L} / \mathrm{S}=20,[\mathrm{Ca}(\mathrm{OH}) 2=20 \mathrm{mM}])$. 


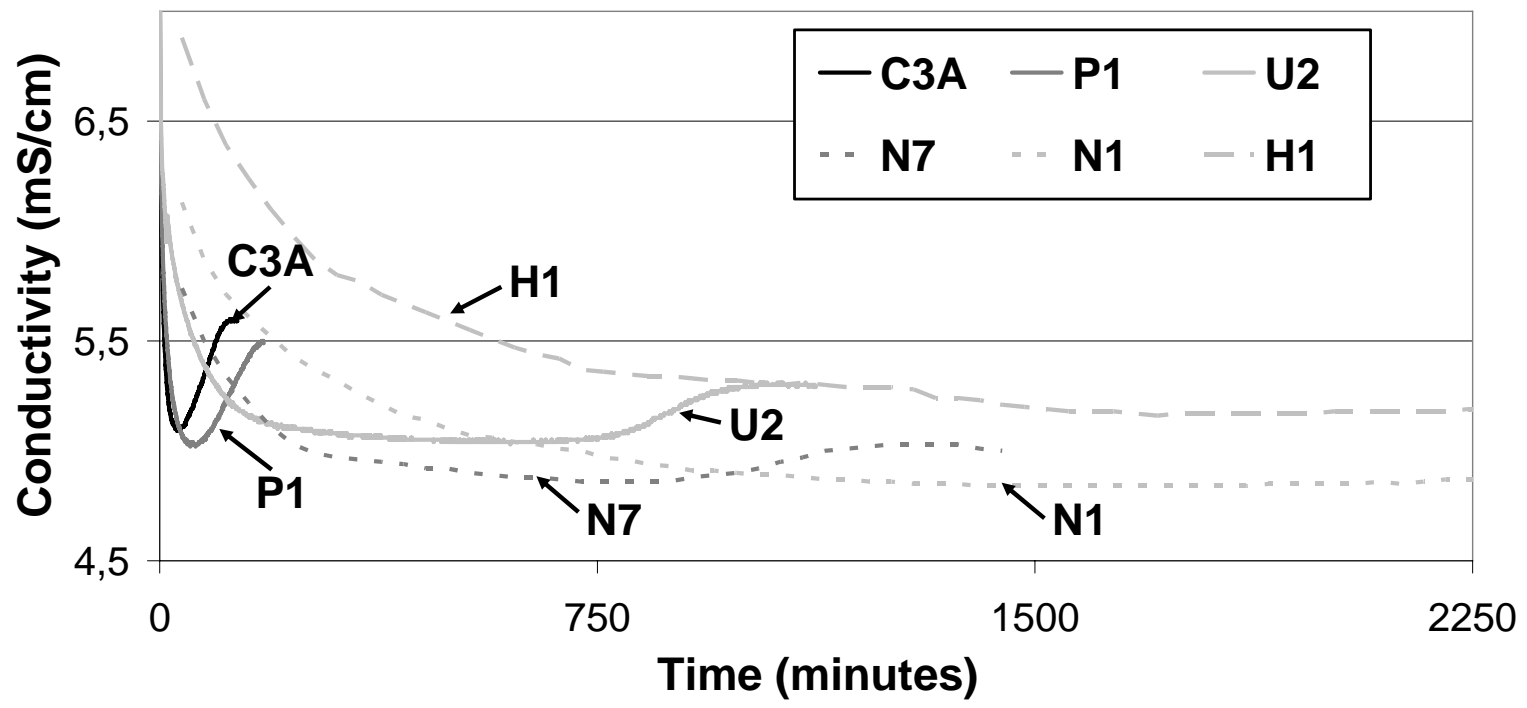

Figure 5. Impact of $\mathrm{CE}$ on $\mathrm{C} 3 \mathrm{~A}$ hydration in limewater without calcium sulphate: conductivity curves $(\mathrm{L} / \mathrm{S}=20,[\mathrm{Ca}(\mathrm{OH}) 2=20 \mathrm{mM}])$.

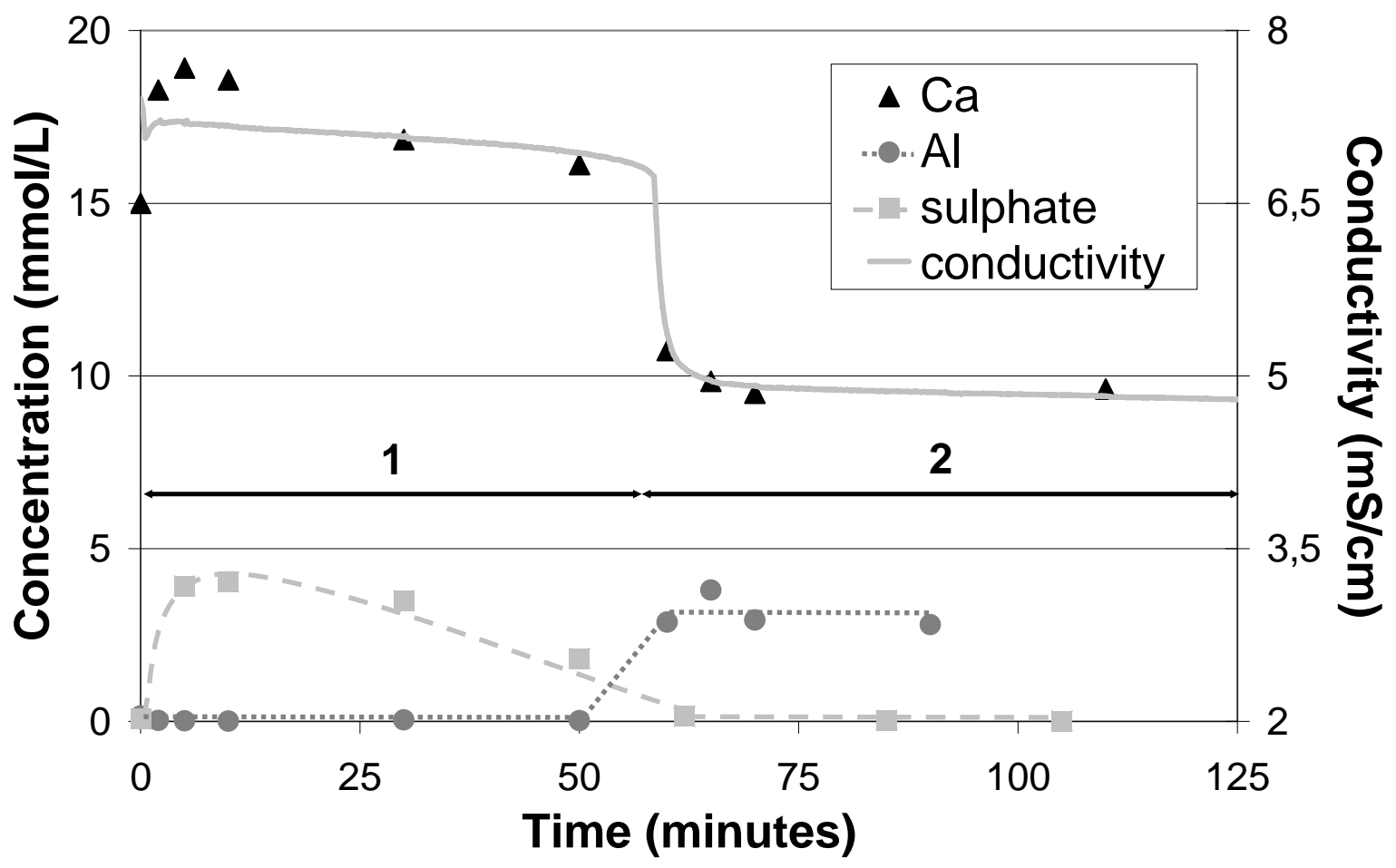

Figure 6. C3A hydration in limewater with calcium sulphate: conductivity curve, calcium, sulphate and aluminium concentration ( $2 \mathrm{wt} \%$ of gypsum, $\mathrm{L} / \mathrm{S}=20,[\mathrm{Ca}(\mathrm{OH}) 2=20 \mathrm{mM}]$ ). 


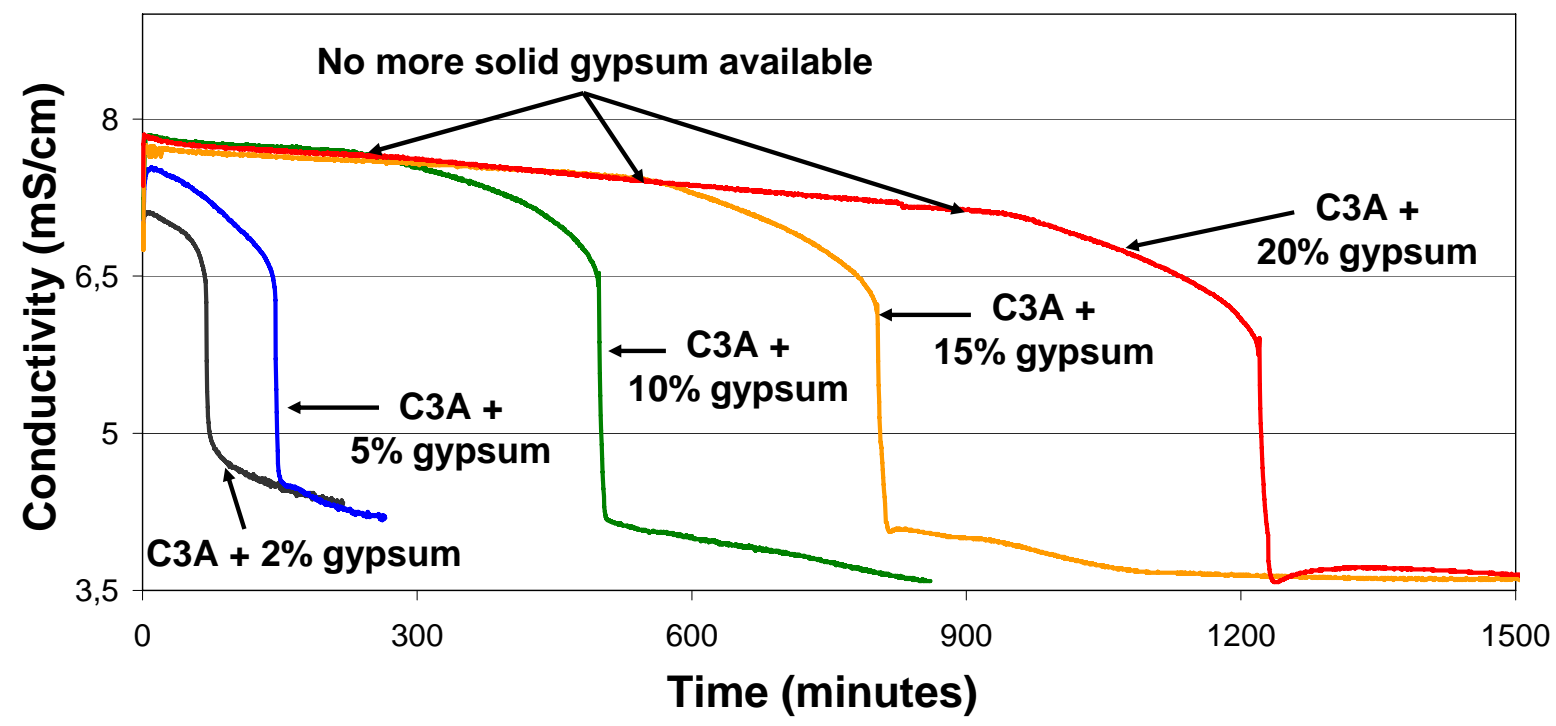

Figure 7. C3A hydration in limewater with various calcium sulphate weight ratio: conductivity curves (various amount of gypsum, $\mathrm{L} / \mathrm{S}=20,[\mathrm{Ca}(\mathrm{OH}) 2=20 \mathrm{mM}]$ ).

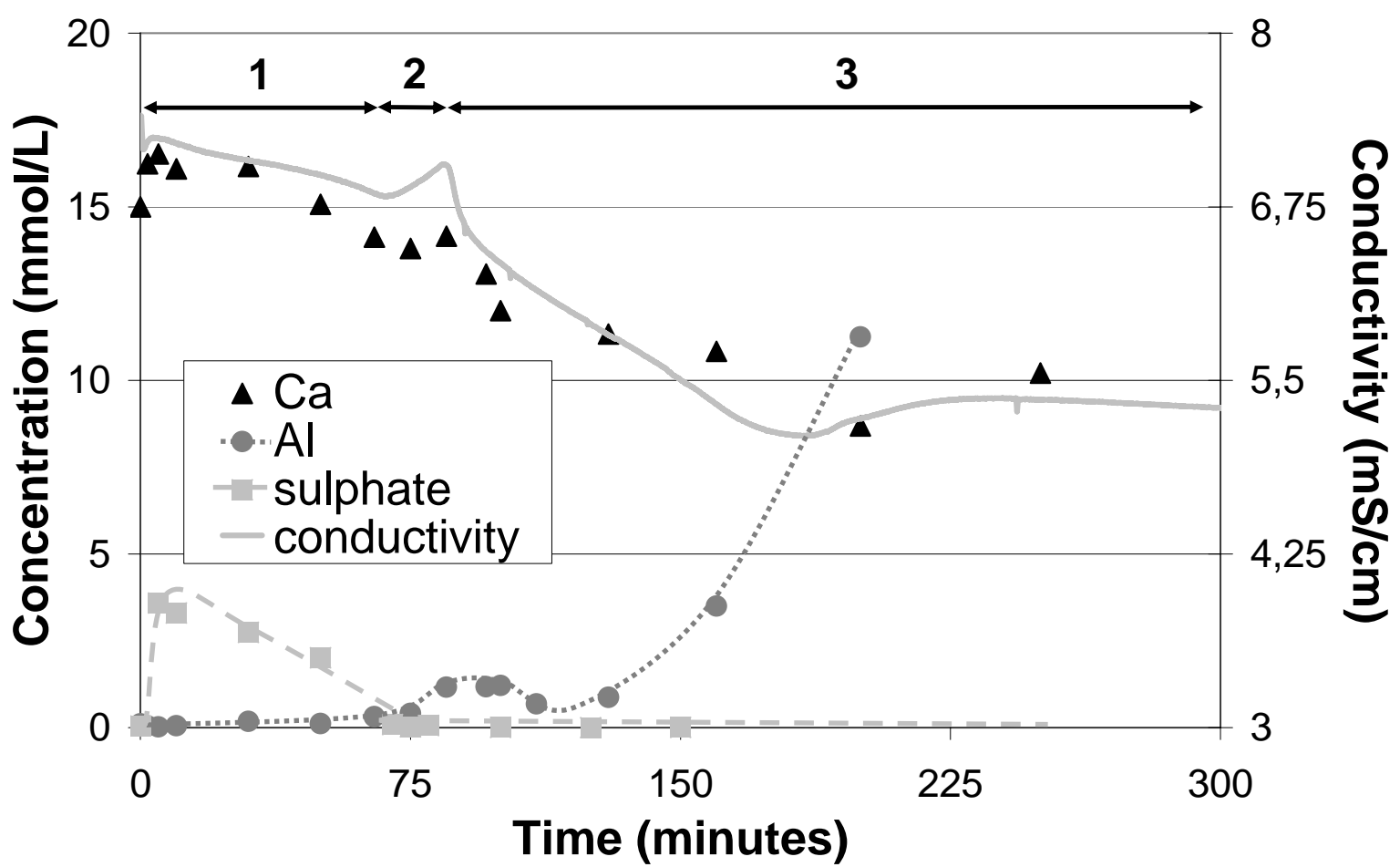

Figure 8. Impact of HEC H1 on C3A hydration in limewater with calcium sulphate: conductivity curve, calcium, sulphate and aluminium concentration ( $2 \mathrm{wt} \%$ of gypsum, L/S $=20$, $[\mathrm{Ca}(\mathrm{OH}) 2=20 \mathrm{mM}])$. 


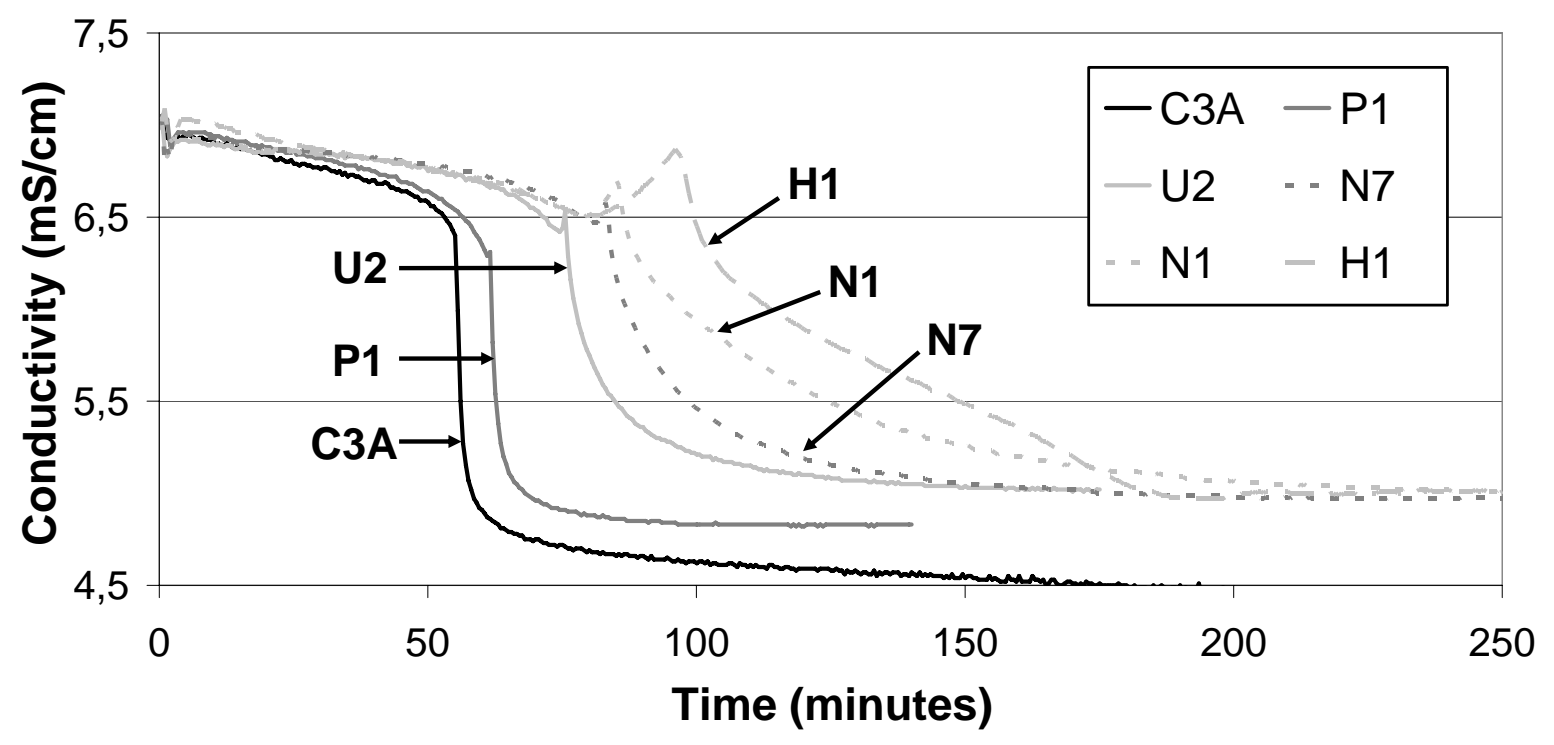

Figure 9. Impact of $\mathrm{CE}$ on $\mathrm{C} 3 \mathrm{~A}$ hydration in limewater with calcium sulphate: conductivity curves (2 wt $\%$ of gypsum, $\mathrm{L} / \mathrm{S}=20,[\mathrm{Ca}(\mathrm{OH}) 2=20 \mathrm{mM}])$.

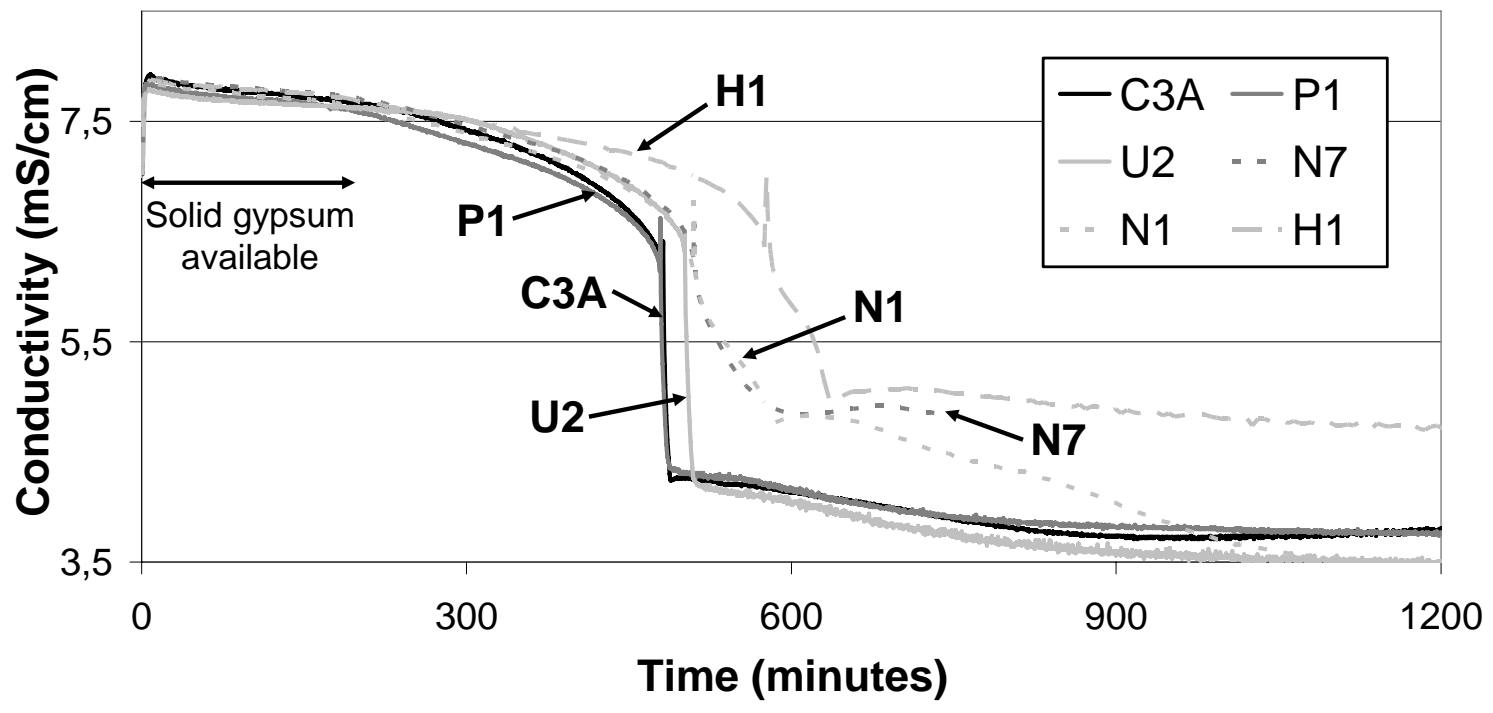

Figure 10. Impact of $\mathrm{CE}$ on C3A hydration in limewater with calcium sulphate: conductivity curves (10 wt $\%$ of gypsum, L/S = 20, [Ca(OH)2=20mM]). 